\title{
Distribution of non-motor symptoms in idiopathic Parkinson's disease and secondary Parkinsonism
}

This article was published in the following Dove Press journal: Journal of Multidisciplinary Healthcare

\author{
Orjan Skogar ${ }^{1,2}$ \\ Mats Nilsson ${ }^{1,3}$ \\ 'FUTURUM, Academy for Health \\ and Care, Region Jönköping County, \\ Jönköping, Sweden; ${ }^{2}$ Institution \\ of Neurobiology, Care Sciences \\ and Society, Karolinska Institute, \\ Stockholm, Sweden; ${ }^{3}$ Department \\ of Medical and Health Sciences, \\ Linköping University, Linköping, \\ Sweden
}

Background: Non-motor symptoms (NMS) are frequent in patients with idiopathic Parkinson's disease (IPD). Clinical expressions, postulated pathophysiological mechanisms, and responsiveness to antiparkinson medication represent differences between IPD and secondary Parkinsonism (SP).

Objective: To evaluate NMS expressions in IPD, SP, and a matched control group.

Methods: The accepted criteria for IPD and SP were controlled for the participants who were consecutively recruited at two outdoor patient clinics. The Well-Being Map ${ }^{\mathrm{TM}}$ was used as the evaluation instrument. These were completed by the participants before their visit. The controls consisted of non-Parkinsonian individuals who were matched by age and gender.

Results: A total of 185 participants participated in the study, IPD/SP/controls; $n=73 / 53$ and 59 , respectively. The mean age was 74 years, and the median duration of disease was $6 / 3$ years. Differences were shown between the combined IPD/SP groups and the controls. Limited differences between the IPD and SP groups could be demonstrated. Symptoms such as pain, decreased taste, as well as sleep and bladder disturbances were more frequent in the IPD group. When more than minor problems with moving were reported, disturbances in sleep and digestion were also noted to a large extent.

Conclusion: Despite differences in the pathophysiological mechanisms between IPD and SP, the study showed only minor differences in the expression of NMS. IPD and SP reported statistically more significant problems in all items compared to the controls. Sleeping problems were strongly associated with symptoms from the gastrointestinal tract, but sleep was only affected by longer disease duration to a minor extent. Motor symptoms, such as morning stiffness, were common in all three groups. Neurodegenerative diseases might have more complex expressions in common than what we have known before and it is certainly not a part of normal aging.

Keywords: idiopathic Parkinson's disease, motor symptoms, non-motor symptoms, secondary Parkinsonism, well-being map, Health related Quality of Life

\section{Introduction}

Idiopathic Parkinson's disease (IPD) is considered a multisystemic neurodegenerative disorder. Age at onset and the course of the disease are heterogeneous between individuals. ${ }^{1}$

Non-motor symptoms (NMS) are recognized as an important part of IPD symptoms and a significant cause of disability and poor health-related quality of life (HRQoL) for patients and caregivers. ${ }^{2}$ In later stages of Parkinsonism, the medical attention of NMS is in a more or less constant focus for patients, families, and people who provide nursing in the care of patients. ${ }^{3}$
Correspondence: Orjan Skogar FUTURUM, Academy for Health and Care, Länssjukhuset Ryhov, SE 55185 Jönköping, Sweden

Tel +4670 55 II 327

Email orjan.skogar@icloud.com 
IPD is the dominant entity in the range of Parkinsonian disorders among patients suffering from disturbances in the control of the locomotor system. Not much is studied and evaluated concerning NMS profiles in patients with other Parkinsonian expressions due to the so-called secondary Parkinsonism (SP). Moreover, a probable hypothesis is that the spectrum of symptoms covered by the questionnaires used for NMS in Parkinsonism may also be common in a senescent general population without locomotor disorders.

The present study was performed with the intentions to clarify similarities and differences of the self-reported prevalence of NMS in IPD, SP, and non-Parkinsonian controls.

The fluctuating NMS profiles can be mapped in different ways. The variations from the time of onset and during the progression of disease are in focus today. With the intention to impede the progression of the disease, the very early, premotor, complex of NMS symptoms is to be identified.

In early prodromal forms of IPD, NMS may include olfactory dysfunction, constipation, depression, and rapid eye movement (REM) sleep behavior disorder. The latter may also appear in the late phases of the disease but has been shown to have a high predictive value before motor symptoms (MS) onset. ${ }^{4} \mathrm{NMS}$ are demonstrated to be prominent in some IPD patients, even during the first years after diagnosis, when dopamine substitution therapy sufficiently controls MS. ${ }^{5}$

During the progression of the disease, NMS comprise a variety of cognitive, neuropsychiatric, sleep, autonomic, and sensory dysfunctions. One hypothesis has been that NMS increase in strength and types over time but the interindividual spectra seem to be huge, and detailed studies on the progression and characteristics of NMS are limited so far. Patterns are heterogeneous, and neuropsychiatric symptoms seem to fluctuate more frequently than others. ${ }^{6}$

HRQoL is often negatively affected by NMS. The reasons for that are numerous as to why we deal with these issues in a more focused way than was common some decades ago.

Pathological structures of synuclein, a small protein, is hypothesized to be of huge importance for the creation of "Lewy bodies" the diagnostic pathoanatomical structures which can be identified within the nigrostriatal system in IPD but Lewy bodies can be seen elsewhere in the brain in neurodegenerative disorders of other types.

NMS is not seldom the first symptoms of IPD. That means that alfa-synucleinopathies->lewy body formation elsewhere in the CNS could possibly explain the clinical expressions of NMS and also why these processes precede the MS's. ${ }^{7,8}$
Pathoanatomical classification of NMS is one way of characterization. The subdivision of NMS into cortical manifestations (cognitive impairment and psychosis), basal ganglia symptoms (apathy, impulse control disorders, and restlessness or akathisia), brainstem symptoms (depression, anxiety, and sleep disorders), and symptoms from other parts of the nervous system, such as sensory disturbances, pain, orthostatic hypotension, and constipation, can be useful. ${ }^{9}$ The accumulation of Lewy body pathology has been described to correlate with NMS expressions as well as the physiological aspects, such as pharmacological contributing factors..$^{10} \mathrm{NMS}$ that fluctuate are called non-motor fluctuations. It is generally accepted that they do not represent non-motor reactions to motor OFF states. They can conceptually be separated from motor fluctuations, despite their frequent temporal co-occurrence. ${ }^{11}$

\section{Classifications of IPD and SP}

Differential diagnosis between the different forms of Parkinsonism is well known to represent a challenge for the clinician. PD diagnosis made in the community by non-experts is associated with a significant error rate. ${ }^{12}$

Changes of symptoms over time must be considered prior to diagnosis. It is of importance to have knowledge of the MDS clinical diagnostic criteria for Parkinson's Disease before diagnosin a patient as IPD. ${ }^{13}$ Probable IPD is diagnosed when bradykinesia and at least one muscular rigidity, rest tremor of 4-6 Hz, and/or postural instability are present. For definite IPD, another three or more of unilateral onset, rest tremor, progressive disorder, excellent levodopa responsiveness, levodopa response over five years, and/or severe levodopa-induced chorea must be present. ${ }^{14}$

IPD can be supported by levodopa or apomorphine tests. Imaging studies such as cranial CT or MRI are helpful to distinguish IPD from atypical or secondary PD. Single-photon emission computed tomography (SPECT) and Positron emission tomography (PET) methods are valuable to distinguish PD tremor from essential tremor (ET) if this is clinically not possible.

One of the most prominent features in SP is either nonlevodopa responsiveness or the lack of more persistent effects of dopamine substitution efforts. ${ }^{15}$ The effects are often described as a transient and nonspecific positive effect on MS and NMS. Increased doses of dopamine replacement therapies often lead to an increase of side effects without a further reduction of symptoms. Of utmost importance to differentiate SP from IPD and atypical Parkinsonism is to recognize whether cerebellar symptoms are present or severe postural instability, 
dyscognitive symptoms, or hallucinations appear within the first years. Freezing of gait during the early course is also a so-called red flag for another diagnosis. ${ }^{16}$ Atypical Parkinsonism is to be considered if severe dysautonomia or disturbances of the locomotor systems in the eyes appear. Other important findings in SP are anamnestic data of repeated stroke or anoxic events with a sudden onset of symptoms. ${ }^{17}$ Previous or ongoing exposure for neuroleptics (drug-induced Parkinsonism [DIP]) is known to be one of the most common reasons for SP. ${ }^{18}$

However, the simultaneous occurrence of some NMS are, in fact, also demonstrated to be helpful in the differentiation between different forms of Parkinsonism. Olfactory dysfunction is shown to be only mildly impaired in atypical Parkinsonism but also in SP. ${ }^{19}$ In the early phases of DIP, more frequent urinary symptoms, decreased attention, and episodes of restless legs symptoms are said to be helpful in the differentiation from IPD. ${ }^{20}$

Patients with ET may have several symptoms of NMS similar to those of IPD. Examples are excessive daytime somnolence and autonomic dysfunction. ${ }^{21}$
Normal pressure hydrocephalus (NPH) is quite a different but also often a treatable cause of SP (shunting). With the triad of gait imbalance, urinary incontinence, and cognitive decline, Parkinsonism may be a presenting phenotype in NPH in up to $70 \%$ of cases. ${ }^{22}$

\section{Self-reported symptoms}

Methods for patients' own registration of NMS symptoms associated with different forms of Parkinsonism are progressing quickly. Web-based solutions for Patients' Own Reporting of symptoms are becoming more established. The In Sweden, The Swedish National Register for neurological diseases, provides this form of technical platform. ${ }^{23}$

Patients' possibilities for answering questions about their self-experienced health and life status before meeting with their care provider at the outdoor clinic have increased. The questions deal with issues such as activities of daily living and HRQoL. By these stances, the intention is for patients to indirectly and directly become more involved in their own care, and caregivers are given the opportunity to study

\section{Parkinson's Well-Being Map ${ }^{\mathrm{TM}}$}

To complete your Parkinson's Well-Being Map ${ }^{\mathrm{TM}}$ refer to steps 1-5 detailed on the previous pages.

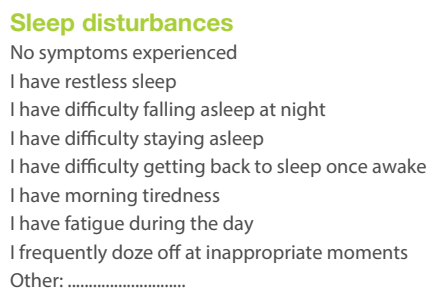

To download additional maps go to www.parkinsons-voices.êt

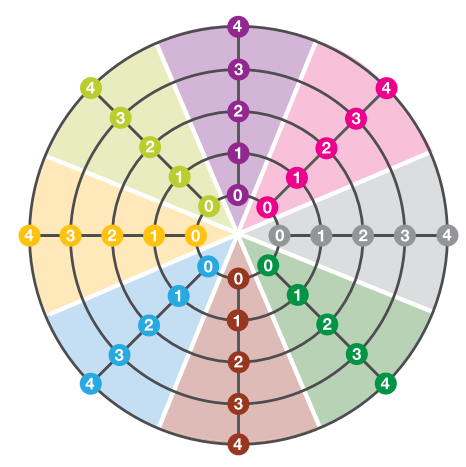

Bladder and Sexual Function No symptoms experienced I feel the urge to pass urine I get up at night to pass urine I have an altered interest in sex I have difficulty having sex Other:
Attention/Memory

No symptoms experienced

I lose my train of thought during conversations am unable to concentrate

have slowness of speech I am forgetful have difficulty remembering names, numbers, events

Other:

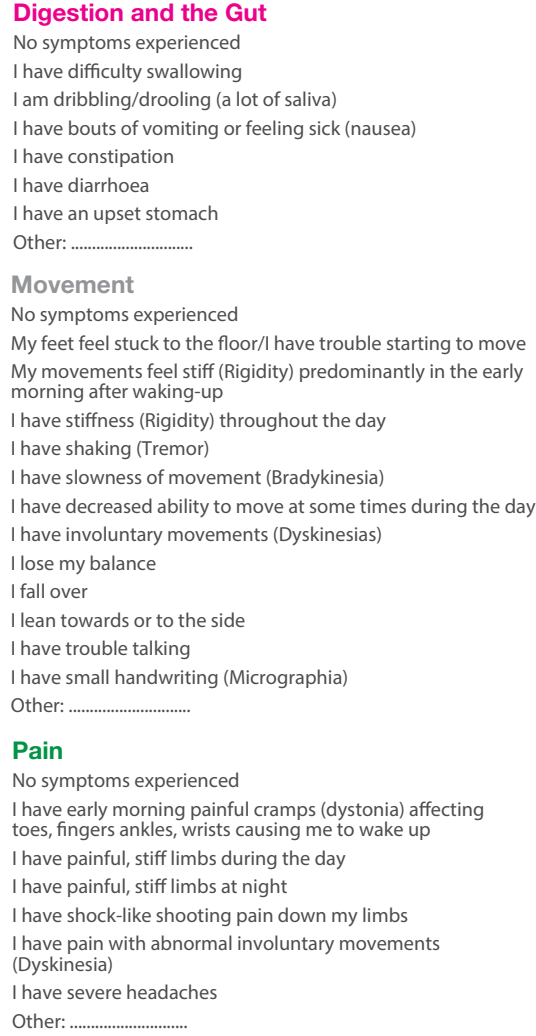

Figure I The Parkinson's Well-Being Map ${ }^{T M}$ (The English version of the WBM ${ }^{T M}$ is a trademark of UCB Pharma S.p.A. C2013 UCB Pharma S.p.A. All rights reserved) categories of symptom complexes illustrated by different colors.

Notes: "No symptoms" are registered as the first state in the columns and illustrated with a zero in the graph. Parkinson's Well-Being Map TM. Available from: http://www. ucb.com/patients/Support-tools/Parkinson-s-Well- Being-Map/well-being-map. ${ }^{24}$ 
a quick and practical visual report and grading of the actual symptoms.

In this study, "The Parkinson's Well-Being Map"TM" was used as a communication tool/check list (Figure 1). This checklist was launched on World Parkinson's Disease Day 2012. It was developed in partnership with the European Parkinson's Disease Association. ${ }^{24}$

The Map is available in several countries and languages. It can be downloaded as a PDF copy of the map to subsequently print and complete by hand. It is also possible to complete it online and save the results to a personal computer or iPhone. The Map enables patients to record and monitor the most common MS and NMS symptoms known from clinical practice and it was created from the results of patients' inquiries. The most prominent symptoms as well as the most important tasks to discuss at the consultation are highlighted through the ordinal grading scale.

Each group of NMS consists of seven to nine specific items, and the issues concerning problems with MS have 13 items. If the patient is free of symptoms, it is recorded in the first row and as zero in the graph. The most prominent symptom in each group is marked and graded in strength from one to four.

\section{Primary and secondary outcomes}

The primary outcome was to measure the NMS and MS symptomatology using the Parkinson's Well-Being Map in the two cohorts (IPD and SP) and to evaluate if differences could be shown between patients diagnosed with IPD and those diagnosed with SP in terms of the type and frequency of symptoms.

Secondary outcomes were to study how the common combinations of NMS appeared within the groups with respect to gender and duration of the disease.

Another secondary outcome was to compare the selfreported NMS in IPD/SP with a non-Parkinsonian control using the same evaluation instrument.

\section{Ethics}

The study was approved by the Regional Ethical Board of Linköping, Sweden, on 20th of April 2016, Dnr 2016/11831. The study followed the Declaration of Helsinki of ethical principles for medical research on humans. The participation in the study was voluntary and the participants could withdraw their participation at any time. All participants provided a written informed consent. ClinicalTrials. gov identifier was NCT03432338 and ID number was Dnr 2016/118-31.

\section{Methods}

Participants diagnosed with IPD or SP were recruited from routine care visits at outpatient departments at two mediumsized county hospitals in southern Sweden. The control group matched by age and gender was recruited from the same area and during the same time period and recruited through local announcements.

Before the start of the study, all patients and, if appropriate, their relatives received careful oral and written instructions of the methodology of the self- reporting "The Well-Being Map" in order to competently fill the map.

The map was sent in paper form by post together with the written call for a revisit set at 1-2 weeks before the visit. The study participants filled the map in advance and brought it back at the visit. Due to the severity of the disease, caregivers were allowed to assist the patient for the completion of the map, if necessary.

All medications were registered and supervised by the study staff. At each site, a trained specialist in movement disorders brought the medical history, examined the patient, and completed other relevant measure tools as defined by the Parkinson Register in National Quality Register. The Unified Parkinson Disease Rating Scale (UPDRS) was used in routine care but was not a part of this study. The maps were then scanned into the journal and registered in a database. For the extraction and processing of data, suitable statistical software for the statistical analysis was used.

\section{Study population}

Inclusion criteria

Patients $>65$ years of age with IPD or SP for more than one year after being diagnosed were invited to participate in the study with the help of convenience sampling. To be classified as IPD, participants fulfilled the clinical criteria for diagnosis according to the UK PDSBBC and signs of SP were excluded.

The SP group was characterized by Parkinsonian symptoms for more than one year but not fulfilling UK PDSBBC. The secondary causes of Parkinsonism were identified and among other criteria characterized by non/partial or transient antiparkinson drugs. Identifiable causes of Parkinsonism were registered. A combination of widespread vascular white matter lesions on MRI and/or CT, lacunar infarcts, and no or only transient response to dopamine replacement therapy were identified. Parkinsonian patients with a history of exposure to neuroleptics and subsequent DIP were identified. 
A control group was recruited, matched by gender and age. This group was recruited via information and oral presentations of the study at local announcements. Comorbidity of another origin was not an exclusion criterion in any group.

\section{Exclusion criteria}

Deficits in the MoCA screening too ${ }^{25}$ with a cutoff at $<26 / 30$ points in any of the three groups.

Patients with symptoms that expressed suspicion of atypical Parkinsonism were excluded for participation in the study. Patients with severe disease of psychiatric origin, atypical Parkinsonism, or severe cognitive impairment were not included in the study.

\section{Statistics}

Chi-squared test and Fisher's exact test were used to analyze comparisons between categorical variables with respect to proportions (presence of different symptoms or characteristics).
When comparing the variables of ordinal data type between different categories, the Mann-Whitney $U$ test was used, and the median with percentiles was presented.

Cutoff was defined as $\geq 2$ on the ordered categorical scale from 0 (no symptoms) to 4 (worst symptoms). The relationships between isolated and combinations of categories were analyzed with respect to odds ratios. The impact of the duration of the diseases and gender was studied.

\section{Results}

A total of 208 participants were recruited to participate in the study (105/103: females/males). Nine patients were excluded due to atypical Parkinsonism and 14 patients due to insufficient data (Figure 2). The mean ages and duration of disease in all groups are shown in Table 1. Six participants were on advanced treatment on apomorphine, Duodopa ${ }^{\mathrm{TM}}$, or Deep Brain Stimulation (3/2/1, respectively). Equipotent levodopa doses and comorbidity of study patients expressed by drug profiles are shown in Table 2, divided according to gender and age of participants.

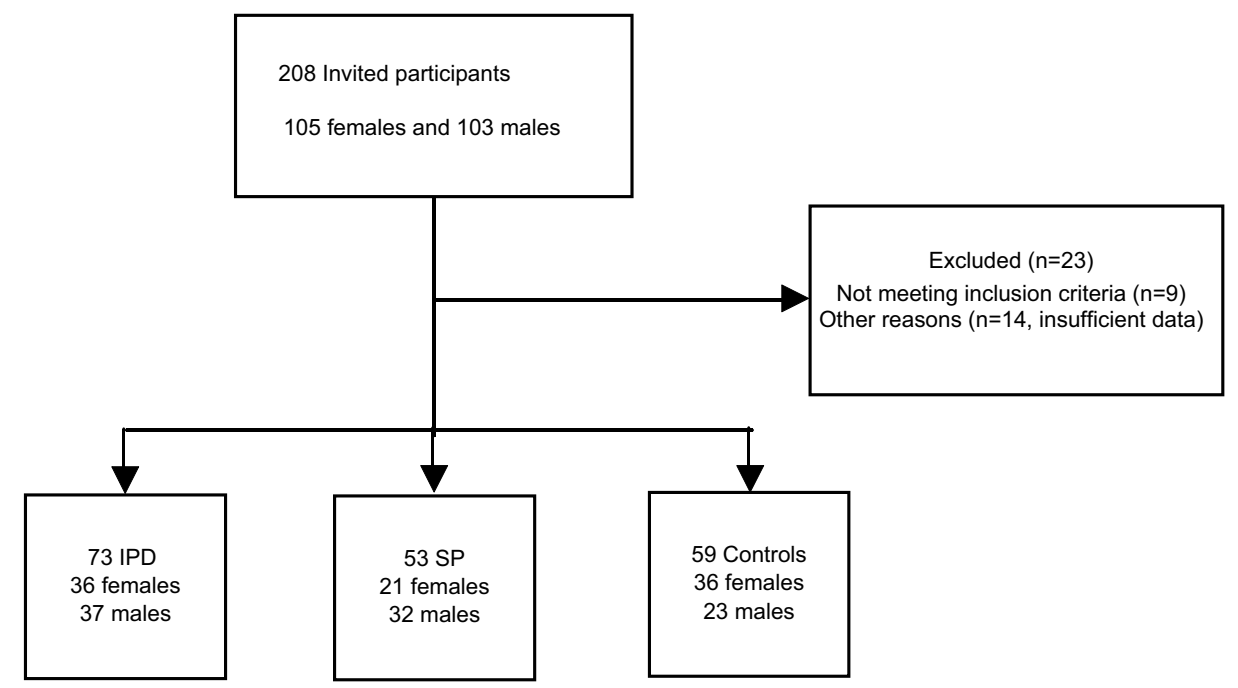

Figure 2 Inclusion and exclusion of participants into the study.

Abbreviations: IPD, idiopathic Parkinson's disease; SP, secondary Parkinsonism.

Table I Characteristics of the study population

\begin{tabular}{lllc}
\hline & $\begin{array}{l}\text { Idiopathic Parkinson's } \\
\text { disease n/mean age in years }\end{array}$ & $\begin{array}{l}\text { Secondary Parkinsonism } \\
\text { n/mean age in years }\end{array}$ & $\begin{array}{l}\text { Total (n) } \\
\text { age in years }\end{array}$ \\
\hline Females & $36 / 74$ & $21 / 70$ & $36 / 78$ \\
Males & $37 / 72$ & $32 / 74$ & $23 / 76$ \\
Total & $73 / 73$ & $53 / 71$ & $59 / 77$ \\
\hline & Duration ${ }^{\text {a }}$ & Duration ${ }^{\mathrm{a}}$ & \\
\hline Total & $6 /(4 / 9)$ & $4 /(2 / 6)$ & - \\
\hline
\end{tabular}

Note: ${ }^{\text {a }}$ alues are given as medians/25th and 75 th percentiles. 


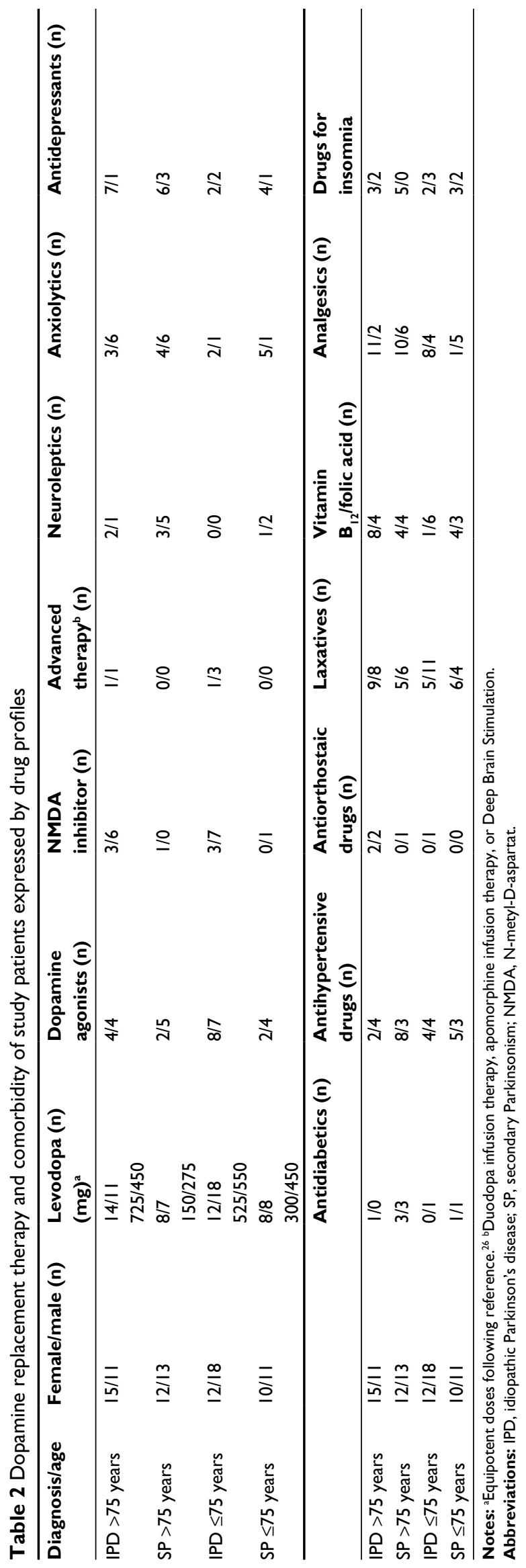

IPD and SP reported statistically significant more problems in all items compared to the controls (Figure 3).

When comparing the IPD and SP groups we found some differences in the reported proportion of several symptoms. The IPD group reported higher prevalence of pain, bladder/sexual function, and sleeping problems than the SP group. Clusters of symptoms (problems with moving, digestion, and sleep; Figure 4) were common in the combined (IPD + SP) groups and increased in prevalence with longer duration of disease.

Neuropsychiatric symptoms were registered in both groups: anxiousness were experienced by 12 and 13 percent, depressive symptoms were experienced by 15 and 14 percent, and fatigue were experienced by 27 and 28 percent of IPD and SP patients, respectively.

Problems with sleep and digestion were the most common reported inconvenience by the IPD and SP patients compared to the controls.

The participants from the IPD and SP groups who reported problems with "digestion and their gut" also reported more problems from the sleeping domain (56 patients vs 14 controls, (cutoff $\geq$ grade 2), Figure 5). Of the patients reporting no problems with digestion, 27 out of 40 also reported sleeping problems $(68 \%)$ and consequently $13 / 40$ (32\%) did not experience any of these NMS.

Of all the participants (IPD/SP groups) who noted $>1$ in strength of "problems with moving", the odds ratio was 5.6 $(1.3-25.0,95 \%$ CI) for the simultaneous presence of "problems with sleep" and "digestion and gut". For pateints with only one additional problem, the corresponding odds ratio was 3.7 (0.9-15.4, 95\% CI).

Morning stiffness as an MS was reported in all the three groups, 45 out of 73 (62 percent) in IPD, 25 out of 53 (47 percent) in SP, and 22 out of 59 (37 percent) in the control group ( $P=0.02$, Fisher's exact test). If only IPD and SP is compared to each other for the presence of morning stiffness, the statistical difference disappears ( $P=0.14$, Fisher's exact test). The average disease duration was seven years for the IPD patients and four years for the SP patients. This difference was statistically significant $(t$-test, $\mathrm{t}[113]=4.3, P<0.0001)$

\section{Discussion}

The nature of chronic progressive neurodegenerative diseases is complex, as are the clinical expressions. The vast majority of patients affected by Parkinsonism are over 60 years of age. This means that comorbidity is usual. The traditional MS of Parkinsonism have been supplemented with a variety of NMS, varying in strength and depending on the severity of disease. Problems with sleep, urinary dysfunction, obstipation, falls, 


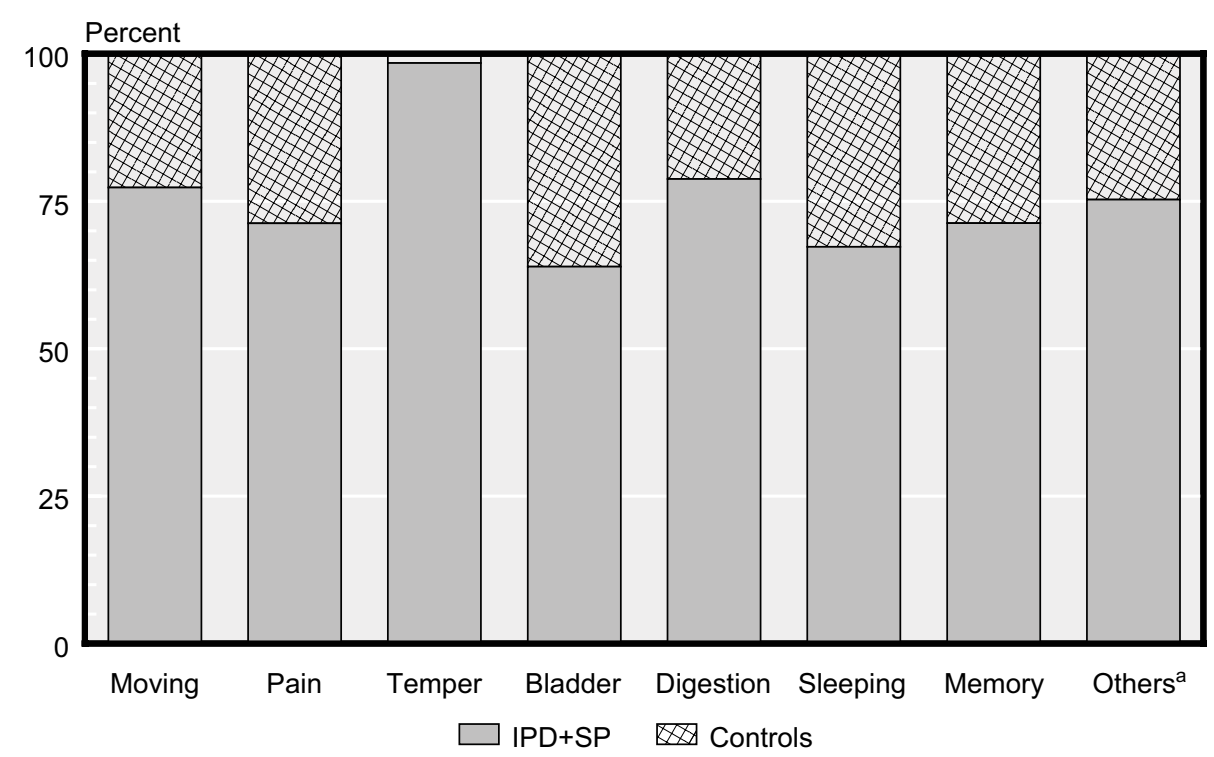

Figure 3 Differences in MS and NMS by group between controls and (IPD + SP).

Note: a ${ }^{2}$ thers = one or more of dizziness, falling, hyposmia/taste, weight loss, increased sweating, and sensitive to stress.

Abbreviations: IPD, idiopathic Parkinson's disease; MS, motor symptoms; NMS, non-motor symptoms; SP, secondary Parkinsonism.

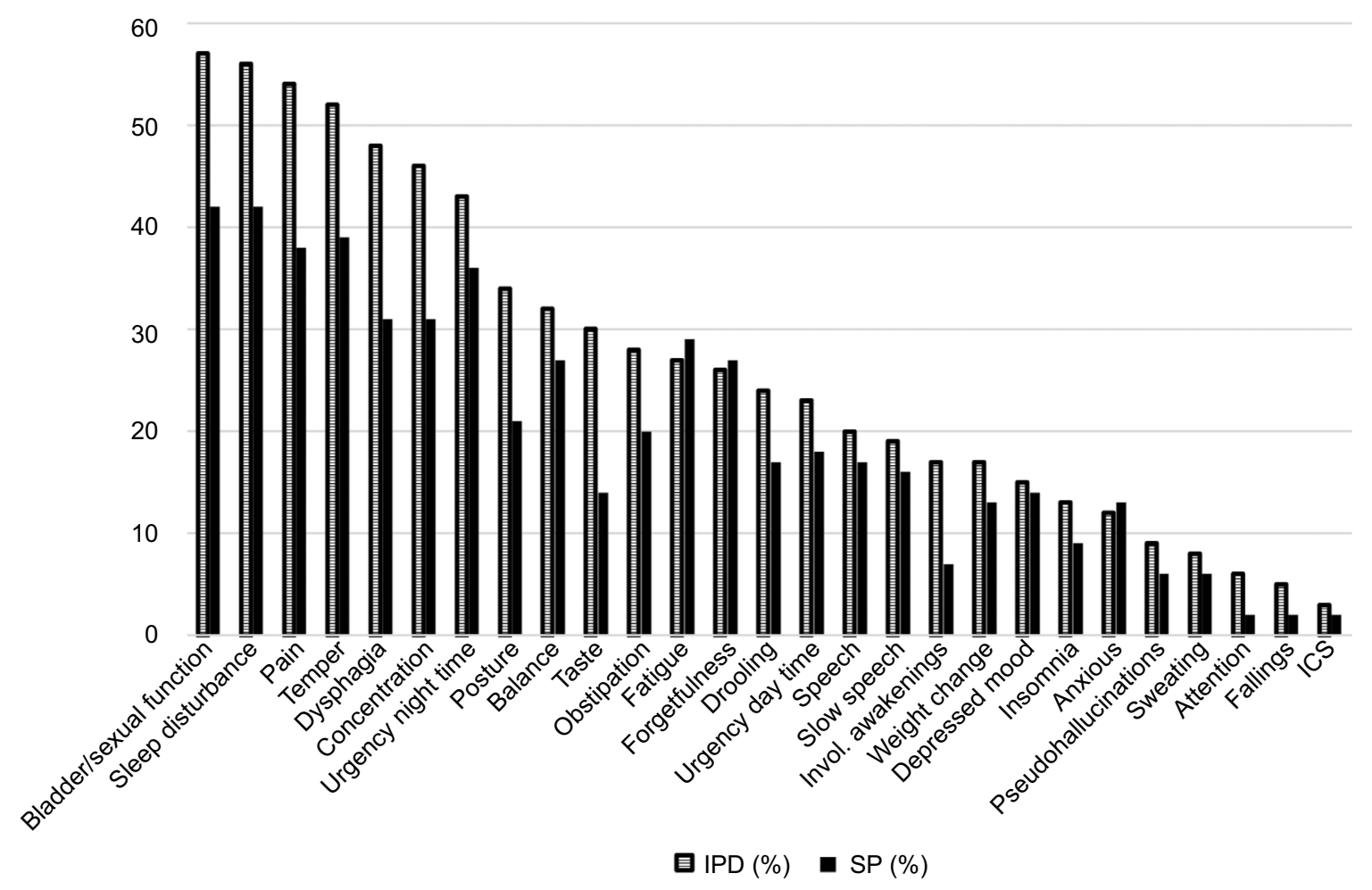

Figure 4 Frequencies of reported clusters symptoms (\%) between IPD and SP groups.

Note: IPD dark staples; SP light staples.

Abbreviations: IPD, idiopathic Parkinson's disease; SP, secondary Parkinsonism; invol., involved; ICS, Impulsive Control Disorder.

and cognitive decline are classical symptoms among IPD and SP but can be assumed to be more common in a senescent population with no other signs of Parkinsonism. This area is insufficiently studied, and in this clinical study, our intention was to compare these symptom expressions between differ- ent groups, one of which was not diagnosed with Parkinsonism. Morning stiffness was reported in all the groups. Pain and stiffness in the morning are common symptoms among individuals with osteoarthritis, a common condition among elderly. 


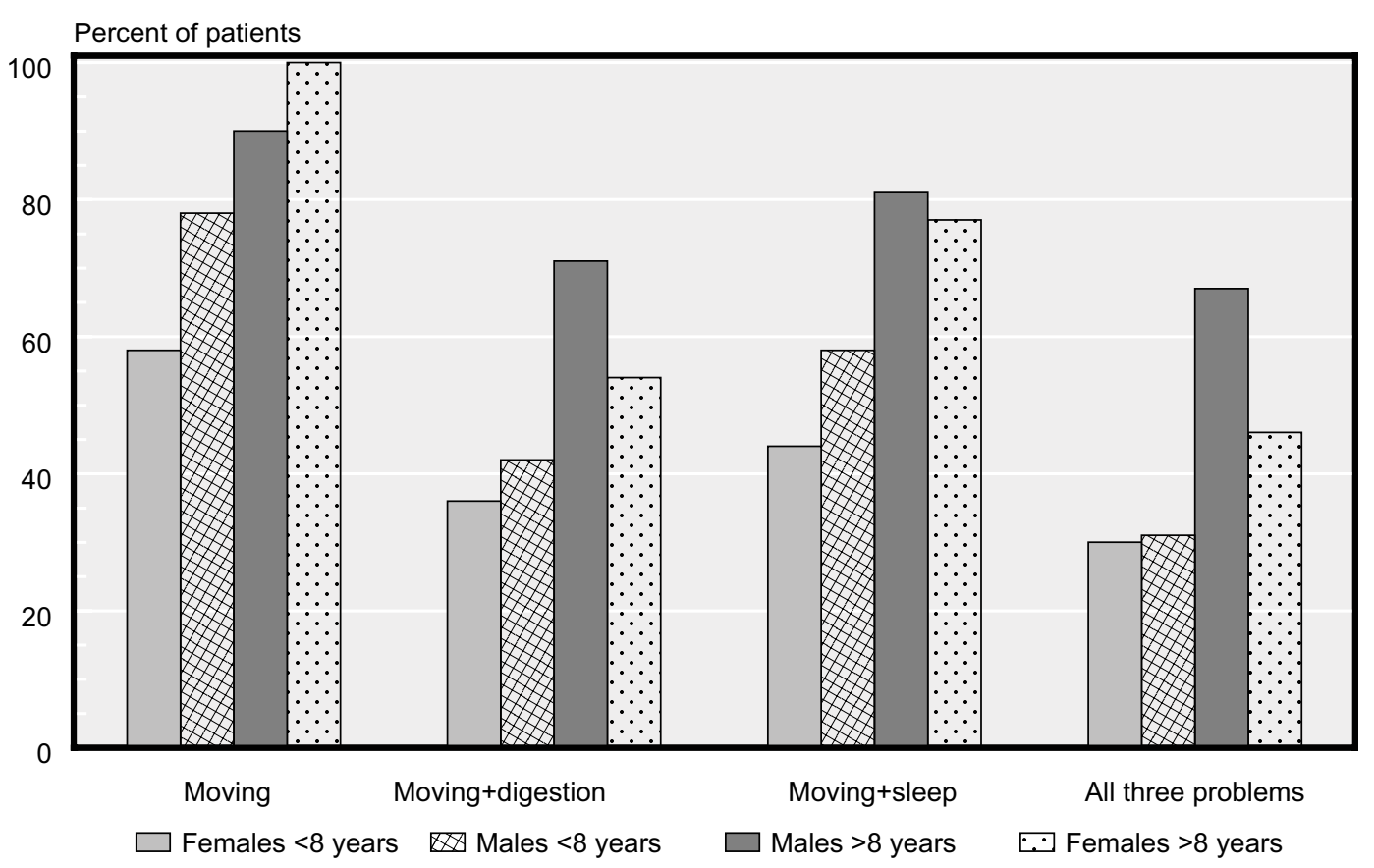

Figure 5 Prevalence in percent for reported problems of moving and/or combined MS and NMS disorders (digestion and/or sleeping problems) in the (IPD + SP) groups split by gender and duration of disease (cutoff $\geq 2$, where $0=$ no symptoms, $4=$ maximal symptoms).

Abbreviations: IPD, idiopathic Parkinson's disease; MS, motor symptoms; NMS, non-motor symptoms; SP, secondary Parkinsonism.

Well-known pathophysiological differences between the groups are the robust data showing that peripheral neuropathy is more common in IPD with long duration of disease as well as autonomic dysfunction, facts that explain common NMS such as pain and bladder/sexual function. ${ }^{27,28}$

It is of utmost importance for the caregiver to be provided with self-experienced data from the patients. The spectrum of symptoms can easily be confusing and lead the clinicians on the wrong track in their attempts to catch the most bothersome symptoms. There are several indications that digital technology will capture parts of the orally communicated medical history. In many places, it is already a part of routine care that patients fill in questionnaires in advance. In this study, we chose to use the Parkinson's Well-Being Map in paper form.

It turned out to be an easy tool for the registration of the most prominent symptoms and soon our team could collect these data that were sorted into well-defined groups of IPD and SP. The pathophysiological background of these entities of Parkinsonism differs and so does the responsiveness to levodopa/dopamine agonists. Numerous clinical differences in MS are described. However, in the present study, we could not identify more prominent differences between NMS expressions in IPD and SP. Symptoms were very common in both groups for known NMS. A hypothesis is that neuronal damage to the dopaminergic pathways, independent of the fundamental cause, has several more or less well-described NMS expressions. A hypothesis is that neuronal damage to the dopaminergic and even other neuronal pathways, independent of the fundamental cause, has NMS expressions of different kinds and strength that extensively exceeds the clinical expressions of neurodegeneration seen in the normal ageing (control group).

For various reasons, NMS are important to study. Early markers for IPD and the subsequent possibilities to affect the progression of disease are a strong focus for researchers. As some NMS severely affect HRQoL negatively and due to the effects of anti-PD medication on NMS, further detailed studies are needed. This and other studies have shown that although they are common symptoms at ages over 60 years, NMS in Parkinsonism outnumber the frequencies among non-Parkinsonians.

\section{Limitations of the study}

\section{Study design limitations (flaws)}

In summary, the diagnostic accuracy for IPD and the symptom complexes that comprise SP can be further improved by using neuroimaging techniques. ${ }^{29,30} \mathrm{~A}$ new approach in this area is electrical neuroimaging in response to olfaction deficit in IPD, which seems to be a promising completion in the diagnostic accuracy. ${ }^{31}$ However, these are not available in 
everyday practice and the clinicians often have to rely on their clinical skills to deal with the diagnostic challenges. A history of cerebrovascular incidents, exposure to neuroleptics, absence of symptoms connected to atypical Parkinsonism, and responsiveness to dopamine replacement therapy were crucial for the diagnose SP.

Participants were recruited from an area in Southern Sweden and all of the participants were Caucasians.

The UPDRS was not registered in this study. NMS are known to fluctuate over time and in intensity. Some NMS varies in expression due to the timepoint of medication intake. In this study, we have not specifically registered if the participants were in the on or off state or the registration of the exact time point of taking the medication in relation to the time point for symptom registration. Neither were possible effects of differences in the duration of exposure of different forms of antiparkinson medication considered for IPD and SP.

There were in fact significant differences in certain items between IPD and SP groups; however, the groups were quite small and it could be vulnerable to underline these findings. Most prominent differences were seen due to duration of disease and the presence of pain, stiffness (females), and problems with sleep (males).

\section{Statistical or data limitations}

A relatively limited numbers of patients from two clinics were recruited. In some cases, participants checked the "no symptoms" checkbox, but when they followed the column downwards, positive symptoms were identified. This incorrect input was taken into consideration when processing the data. The presence of symptoms was interpreted as the correct answer in these cases.

\section{Conclusion}

This study clearly shows statistically more NMS among elderly patients with IPD or SP compared to controls of the same age. Certain clusters of symptoms seem to be common among IPD and SP patients.

There was an increased reporting of some, but not all, NMS symptoms with increasing duration of the diseases. Self-reported problems with moving were associated with a more frequent notification of problems with digestion and sleep. Trends toward more memory problems were seen in the SP group compared to the IPD group. Morning stiffness was common in all the groups but dominated in the IPD group.

The Parkinson's Well-Being Map proved to be an easy checklist to complete by most participants. Examining doctors experienced facilitation for focusing on the most prominent symptoms.

Each patient with either IPD or SP have different profiles of expression of their symptoms, all symptoms expressed in the check list may be found in parkinsonian patients but the individual pattern is different. Clinicians are in need of a screening instrument that permits a quick view of the actual self-experienced dominating NMS and MS.

\section{Acknowledgments}

Dr Skogar would like to thank the participants in this study and the Parkinson Team at County Hospital Ryhov, Jonkoping, and at Hoglandssjukhuset, Eksjo for their assistance. This research received financial support through a grant from the commercial sector (UCB Pharma) for the language correction and publication costs.

\section{Author contributions}

All authors contributed toward data analysis, drafting and critically revising the paper, gave final approval of the version to be published, and agree to be accountable for all aspects of the work.

\section{Disclosure}

The authors report no conflicts of interest in this work.

\section{References}

1. Abdullah R, Basak I, Patil KS, Alves G, Larsen JP, Møller SG. Parkinson's disease and age: The obvious but largely unexplored link. Exp Gerontol. 2015;68:33-38.

2. Prakash KM, Nadkarni NV, Lye WK, Yong MH, Tan EK. The impact of non-motor symptoms on the quality of life of Parkinson's disease patients: a longitudinal study. Eur J Neurol. 2016;23(5):854-860.

3. Mosley PE, Moodie R, Dissanayaka N. Caregiver Burden in Parkinson Disease: A Critical Review of Recent Literature. J Geriatr Psychiatry Neurol. 2017;30(5):235-252.

4. Mahlknecht P, Seppi K, Poewe W. The Concept of Prodromal Parkinson's Disease. J Parkinsons Dis. 2015;5(4):681-697.

5. Erro R, Picillo M, Vitale C, et al. The non-motor side of the honeymoon period of Parkinson's disease and its relationship with quality of life: a 4-year longitudinal study. Eur J Neurol. 2016;23(11):1673-1679.

6. Storch A, Schneider CB, Wolz M, et al. Nonmotor fluctuations in Parkinson disease: severity and correlation with motor complications. Neurology. 2013;80(9):800-809.

7. Jellinger KA. Neuropathobiology of non-motor symptoms in Parkinson disease. J Neural Transm. 2015;122(10):1429-1440.

8. Titova N, Chaudhuri KR. Personalized medicine in Parkinson's disease: Time to be precise. Mov Disord. 2017;32(8):1147-1154.

9. Bayulkem K, Lopez G. Nonmotor fluctuations in Parkinson's disease: clinical spectrum and classification. J Neurol Sci. 2010;289(1-2):89-92.

10. Lee HM, Koh SB. Many Faces of Parkinson's Disease: Non-Motor Symptoms of Parkinson's Disease. J Mov Disord. 2015;8(2): 92-97.

11. Classen J, Koschel J, Oehlwein C, et al. Nonmotor fluctuations: phenotypes, pathophysiology, management, and open issues. J Neural Transm. 2017;124(8):1029-1036. 
12. Brooks DJ. Parkinson's disease: diagnosis. Parkinsonism Relat Disord. 2012;18(Suppl 1):S31-S33.

13. Postuma RB, Berg D, Stern M, et al. MDS clinical diagnostic criteria for Parkinson's disease. Mov Disord. 2015;30(12):1591-1601.

14. Hughes AJ, Daniel SE, Kilford L, Lees AJ. Accuracy of clinical diagnosis of idiopathic Parkinson's disease: a clinico-pathological study of 100 cases. J Neurol Neurosurg Psychiatry. 1992;55(3):181-184.

15. Korczyn AD. Vascular parkinsonism--characteristics, pathogenesis and treatment. Nat Rev Neurol. 2015;11(6):319-326.

16. Christine CW, Aminoff MJ. Clinical differentiation of parkinsonian syndromes: prognostic and therapeutic relevance. Am J Med. 2004;117(6):412-419.

17. Defebvre L, Krystkowiak P. Movement disorders and stroke. Rev Neurol. 2016;172(8-9):483-487.

18. Blanchet PJ. Antipsychotic drug-induced movement disorders. Can J Neurol Sci. 2003;30(Suppl 1):S101-S107.

19. Müller A, Müngersdorf M, Reichmann H, Strehle G, Hummel T. Olfactory function in Parkinsonian syndromes. J Clin Neurosci. 2002;9(5):521-524.

20. Kim JS, Youn J, Shin H, Cho JW. Nonmotor symptoms in drug-induced parkinsonism and drug-naïve Parkinson disease. Can J Neurol Sci. 2013;40(1):36-41.

21. Lee SM, Kim M, Lee HM, Kwon KY, Koh SB. Nonmotor symptoms in essential tremor: Comparison with Parkinson's disease and normal control. J Neurol Sci. 2015;349(1-2):168-173.

22. Keener AM, Bordelon YM. Parkinsonism. Semin Neurol. 2016;36(4): 330-334.
23. Hillert J, Stawiarz L. The Swedish MS registry - clinical support tool and scientific resource. Acta Neurol Scand. 2015;132(199):11-19.

24. Parkinson's Well-Being Map ${ }^{\mathrm{TM}}$. Available from:http://www.ucb.com/ patients/Support-tools/Parkinson-s-Well-Being-Map/well-beingmap. Accessed September 19, 2018.

25. Chou KL, Lenhart A, Koeppe RA, Bohnen NI. Abnormal MoCA and normal range MMSE scores in Parkinson disease without dementia: cognitive and neurochemical correlates. Parkinsonism Relat Disord. 2014;20(10):1076-1080.

26. Tomlinson CL, Stowe R, Patel S, Rick C, Gray R, Clarke CE. Systematic review of levodopa dose equivalency reporting in Parkinson's disease. Mov Disord. 2010;25(15):2649-2653.

27. Zis P, Grunewald RA, Chaudhuri RK, Hadjivassiliou M. Peripheral neuropathy in idiopathic Parkinson's disease: A systematic review. $J$ Neurol Sci. 2017;378:204-209.

28. Pfeiffer RF. Autonomic dysfunction in Parkinson's disease. Expert Rev Neurother. 2012;12(6):697-706.

29. Shafieesabet A, Fereshtehnejad SM, Shafieesabet A, et al. Hyperechogenicity of substantia nigra for differential diagnosis of Parkinson's disease: A meta-analysis. Parkinsonism Relat Disord. 2017; 42:1-11.

30. Politis M. Neuroimaging in Parkinson disease: from research setting to clinical practice. Nat Rev Neurol. 2014;10(12):708-722.

31. Prashanth R, Dutta Roy S, Mandal PK, Ghosh S. High-Accuracy Detection of Early Parkinson's Disease through Multimodal Features and Machine Learning. Int J Med Inform. 2016;90:13-21.
Journal of Multidisciplinary Healthcare

\section{Publish your work in this journal}

The Journal of Multidisciplinary Healthcare is an international, peerreviewed open-access journal that aims to represent and publish research in healthcare areas delivered by practitioners of different disciplines. This includes studies and reviews conducted by multidisciplinary teams as well as research which evaluates the results or conduct of such teams or health

\section{Dovepress}

care processes in general. The journal covers a very wide range of areas and welcomes submissions from practitioners at all levels, from all over the world. The manuscript management system is completely online and includes a very quick and fair peer-review system. Visit http://www.dovepress.com/ testimonials.php to read real quotes from published authors. 